\title{
Estimates of the Trajectory Parameters and Thermal Loads for a Ballistic Capsule Returning from the Moon with Multiple Dives into the Earth Atmosphere
}

\author{
${ }^{1}$ Victor V. Leonov and ${ }^{2}$ Dmitriy A. Grishko \\ ${ }^{1}$ Department of Spacecrafts and Launch Vehicles, \\ ${ }^{2}$ Department of Theoretical Mechanics, Bauman Moscow State Technical University, \\ Russia 5, 2nd Baumanskaya St., 105005 Moscow, Russia
}

\begin{abstract}
Numerical analysis of an approximate return trajectory from the Moon with multiple dives into the atmosphere is carried out. The motion which is assumed to be planar is modeled in a central gravity field. The target perigee altitude is a variate for the first dive into the atmosphere. The descent vehicle is a ballistic capsule with zero lift-drag. The target perigee altitude is shown as having an effect on the number of dives into the atmosphere and on the apogee altitude of the orbit formed after a successive penetration into the atmosphere. Estimates are given for the thermal loads on the vehicle in the dense atmosphere and for the mass of the ablative thermal protection entrained from the space vehicle surface. Feasibility of such trajectories is shown, their advantages and disadvantages are discussed.
\end{abstract}

Key words: Moon exploration, return trajectory, multiple dives into the atmosphere, ballistic capsule, descent module, ablative thermal protection system

\section{INTRODUCTION}

At present, manned Lunar missions with landing to its surface were realized only in 1969-1972 in Apollo programs. In these missions, samples of Lunar rocks and soil were gathered, retroreflectors were installed on the Moon surface (after which the accuracy of measurement of the Earth-to-Moon distance was significantly improved), the effect of lunar conditions on human beings and equipment was analyzed and a number of research studies was carried out (Lyndon, 1975). Unfortunately, the Apollo program and later the Soviet Lunar exploration program were closed, delaying for a long time studies of the Moon.

During the past decade there has been a renewed interest on Lunar studies which on the practical side included the Japanese Kaguya Selenological and Engineering Explorer, the American LRO and LCROSS missions (Keller et al., 2016), the Chinese Chang'e exploration program and the Indian Chandrayan exploration program (Jin et al., 2013). There are also plans of long-haul Lunar developments which involve in particular, delivery of various cargos back to the Earth (Murtazin, 2014; Grabois, 2011). For this purpose, use can be made of special transfer modules, whose design layout depends on the type of delivered cargos. A recovery Ballistic Capsule (BC) which features zero lift-drag in the atmosphere is a simplest example of a module of this kind. In general, such a $\mathrm{BC}$ has no attitude control but there is also an option to equip it with an attitude control thruster unit with vernier engines installed for correct positioning of the propellant system. In the problem of $\mathrm{BC}$ recovery of great value is the accuracy of maneuvers: both for those which transfer the $\mathrm{BC}$ into the Moon-Earth trajectory and those that adjust this trajectory. Execution errors in the velocity impulse can be not that high for the BC to miss the Earth but still they can be sufficient to lead to an off-nominal descent which cannot be made nominal with the available propulsion system.

In the present study, we give the analysis findings for motion trajectories and thermal loads for a $\mathrm{BC}$ approaching the Earth with off-nominal parameters of the trajectory for which multiple dives into the atmosphere are possible. The velocity of the $\mathrm{BC}$ on the atmosphere entry boundary is close to the local parabolic velocity which may result in two opposite effects. On the one hand, a BC should be exposed to lesser thermal loads due to a more gradual trajectory. In addition to this, its velocity decreases gradually on each atmospheric path (as opposite to its decelerating to zero in 7-12 $\mathrm{min}$ for a direct landing). On the other hand, the total action of such thermal loads is distributed over a more lengthy time interval which may result in burn-outs of the thermal

Corresponding Author: Victor V. Leonov, Department of Spacecrafts and Launch Vehicles, 
protection coating and in the destruction of the $\mathrm{BC}$. The following problems were considered in the analysis: estimate of the minimal perigee altitude for which the $\mathrm{BC}$ trajectory apogee will not exceed $200 \mathrm{~km}$ due to strong aerodynamic breaking in the atmosphere which results in an exit from the orbit during the next orbit pass; estimation of $\mathrm{BC}$ aerobraking duration with various perigee altitudes of the return trajectory; estimate of the thermal loads acting on the $\mathrm{BC}$ while it passes through the dense atmosphere; estimate of the mass of the ablative thermal protection entrained from the space vehicle's surface.

\section{MATERIALS AND METHODS}

Simplified mathematical model of motion: The BC under consideration weighs $5500 \mathrm{~kg}$, its form is close to a sphere of diameter $3.9 \mathrm{~m}$ which partially corresponds to the parameters of the Apollo command module. Since, the BC has zero lift-drag, it can be considered in the first approximation as a material point subject to the force of Earth's gravity and the retarding force of the atmosphere. It is assumed that at the initial moment the $\mathrm{BC}$ crosses the Moon's sphere of influence and in the first approximation lies on a Hohmann ellipse (Hohmann, 1925) whose apogee is $100 \mathrm{~km}$ higher than the mean distance between Earth and the Moon and whose perigee is a variate (Fig. 1). The further trajectory is flat with respect to Earth, the atmosphere is taken into consideration up to $1500 \mathrm{~km}$. The mathematical model of such a motion reads as:

$$
\begin{gathered}
\dot{V}_{x}=-\frac{\mu}{r^{3}} x-C_{x a} \cdot \frac{\rho V^{2}}{2 m} S_{m} \cdot \frac{V_{x}}{V} \\
\dot{V}_{y}=-\frac{\mu}{r^{3}} y-C_{x a} \cdot \frac{\rho V^{2}}{2 m} S_{m} \cdot \frac{V_{y}}{V} \\
\dot{x}=V_{x} \\
\dot{y}=V_{y}
\end{gathered}
$$

Equations 1-4 are written in the inertial frame with origin at the Earth center, $r=\sqrt{x^{2}+y^{2}}$ is the length of the $\mathrm{BC}$ radius vector, $\mu=398600.44 \mathrm{~km}^{3} / \mathrm{s}^{2}$ is the geocentric gravity constant, $\mathrm{V}=\sqrt{\mathrm{V}_{\mathrm{x}}^{2}+\mathrm{V}_{\mathrm{y}}^{2}}$ is the modulus of the $\mathrm{BC}$ velocity, $\mathrm{C}_{\mathrm{za}}$ is the aerodynamic drag force coefficient, $\rho$ is the atmosphere density according to GOST $P$ 25645.166-2004 (altitudes 120-1500 km) and GOST 4401-81 (altitudes 0-120 km), $\mathrm{S}_{\mathrm{m}}$ is the area of the descent modulus mid-section. The above system of equations was integrated numerically using the fourth-order Runge-Kutta method with constant step.

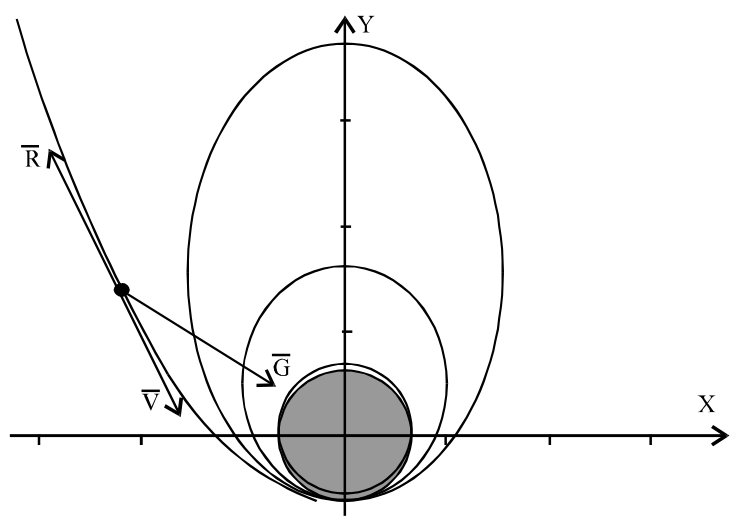

Fig. 1: The qualitative shape of the BC trajectory

The employed mathematical motion model involves a considerable number of assumptions and simplifications: motion is assumed to be two-dimensional, no consideration is taken of the noncentrality of the gravitational field. The real trajectory of a $\mathrm{BC}$ with respect to Earth on the Moon's sphere of influence will be different from an ellipse and the distance from Earth to Moon varies with time. On the other hand, our solution to the problem is superficial to a certain extent and so, due to the uncertainty in the initial conditions for the backhaul start from the Moon, all these simplifications are considered quite acceptable.

\section{Influence analysis of the atmosphere on the $\mathrm{BC}$ return} trajectory: The perigee of the $\mathrm{BC}$ return trajectory lies in the dense atmosphere. The intensity of atmospheric deceleration of the descent module is different depending on the selected perigee altitude because the density does not depend linearly on the altitude. This gives us different apogee altitudes of the ellipse obtained after the first penetration into the atmosphere: the larger the perigee of the return trajectory, the larger is the apogee of the so-formed ellipse. The BC will stay at the near-Earth orbit until, during a consecutive passage of the perigee region, its apogee will also lie in the dense atmosphere. The apogee decreases several times faster than the perigee during the atmospheric deceleration. If the apogee was $200 \mathrm{~km}$ on the pervious orbit pass, then, considering the small perigee altitude, the $\mathrm{BC}$ will fall back to Earth during the next atmosphere penetration.

There also exists a perigee altitude for which the apogee of the orbit formed after the first penetration into the atmosphere directly reaches the target value of $200 \mathrm{~km}$ (Fig. 2). This altitude is approximately $66.5 \mathrm{~km}$ for the above descent module. This trajectory corresponds to the smallest flight time and the greatest thermal loads acting on the descent module. 
J. Eng. Applied Sci., 14 (6): 1775-1780, 2019

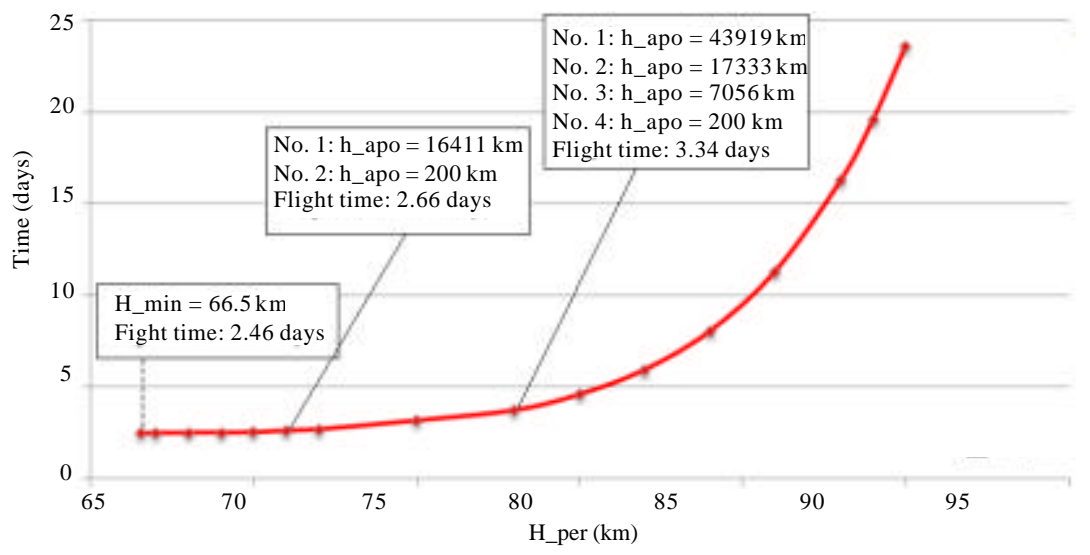

Fig. 2: Flight time to achieve $200 \mathrm{~km}$ apogee altitude versus the perigee altitude of the return trajectory

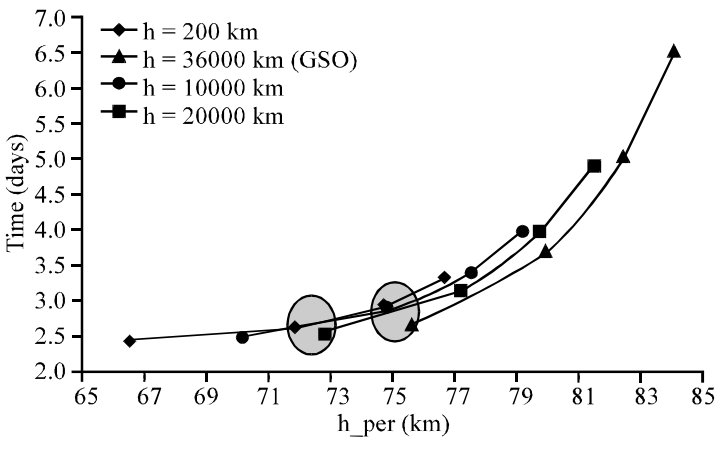

Fig. 3: Flight time duration versus the perigee altitude of the return trajectory for various target apogee altitudes attained with various dives into the atmosphere

The time to achieve the apogee altitude of $200 \mathrm{~km}$ varies nonuniformly with increasing the perigee altitude. The increase by $71 \mathrm{~km}$ of the perigee altitude of $66.5 \mathrm{~km}$ results in another penetration into the atmosphere; another increase by $5-7 \mathrm{~km}$ gives dives whose multiplicity is divisible by 4 . A further increase in the perigee altitude of the return trajectory to $90 \mathrm{~km}$ may result in multiple dives into the atmosphere that increases drastically the flight duration and sends the space vehicle far beyond the GSO altitude for few first revolutions.

Figure 3 shows the flight time versus the altitude of the perigee of the return trajectory. Each curve corresponds to a specific target apogee altitude; The points on this curve correspond to the number of penetrations into the atmosphere to achieve the target apogee altitude.

Figure 3 shows the rhombic makers show the flight duration corresponding to an orbit with apogee altitude $200 \mathrm{~km}$. It is seen that when the perigee altitude of the return trajectory is approximately $72 \mathrm{~km}$ one can either obtain $200 \mathrm{~km}$ apogee altitude with two fold penetration into the atmosphere or $\sim 20000 \mathrm{~km}$ apogee altitude with a single atmosphere penetration. Similarly, for the target perigee altitude of $75 \mathrm{~km}$, the attainment of the apogee altitude of $200 \mathrm{~km}$ with three penetrations into the atmosphere is equivalent to the time required to reach a geostationary Earth orbit immediately after the first penetration.

\section{RESULTS AND DISCUSSION}

Analysis of thermal loads: A space vehicle entering a planet atmosphere is a subject to considerable thermal loads, whose magnitude depends on its form and sizes, the atmosphere entry parameters and the descent profile. For ballistic spherical descent modules there exists a number of empirical dependences from which one can estimate with sufficient accuracy the thermal loads on the forward hemisphere (which is the most heat loaded part of the space vehicle (Avduevskii, 1992; Nikitin, 2006).

The convective heat flux density at the critical point of the spherical blunt nose (the forward hemisphere) with laminar flow can be obtained from the dependence:

$$
\mathrm{q}_{\mathrm{w}, 1}=\frac{1,318 \cdot 10^{5}}{\sqrt{\mathrm{R}}}\left(\frac{\rho}{\rho_{0}}\right)^{0.5}\left(\frac{\mathrm{V}}{\mathrm{V}_{1}}\right)^{3.25}\left[\frac{\mathrm{kW}}{\mathrm{m}^{2}}\right]
$$

where, $R$ is the radius of the sphere, $\rho$ and $\rho_{0}$ are respectively, the atmosphere density at flight trajectory and near the Earth, $V$ and $V_{1}$ are respectively, the flight velocity and the first cosmic velocity. Similarly, for the turbulent regime:

$$
\mathrm{q}_{\mathrm{w}, \mathrm{t}}=1.15 \cdot 10^{6} \frac{\rho^{0.8}}{\mathrm{R}^{0.2}}\left(\frac{\mathrm{V}}{\mathrm{V}_{1}}\right)^{3.19}\left[\frac{\mathrm{kW}}{\mathrm{m}^{2}}\right]
$$

The Reynolds number: 


$$
\mathrm{Re}=\frac{\mathrm{V} \cdot \mathrm{R}}{v}=\frac{\rho \cdot \mathrm{V} \cdot \mathrm{R}}{\mu}
$$

serves as a criterion for assessing the transition from a laminar flux in the boundary layer to the turbulent regime. Here, $\mu$ is the coefficient of dynamic viscosity and $\mu$ is the coefficient of kinematic viscosity. The Reynolds number $\operatorname{Re}=10^{6}$ is considered to correspond to the change of regimes of the flow. The regime becomes turbulent when this value is exceeded.

To determine the convective heat flux density at an arbitrary point of the forward hemisphere (in the two-dimensional setting of the problem) the following formulas can be used (Avduevskii, 1992):

$$
\begin{aligned}
& \mathrm{q}_{\mathrm{w}, 1}(\Omega)=\mathrm{q}_{\mathrm{w}, 1}^{0}-\sin ^{2} \Omega \\
& \mathrm{q}_{\mathrm{w}, \mathrm{t}}(\Omega)=1.04-\mathrm{q}_{\mathrm{w}, \mathrm{t}}^{0} \cdot\left(\sin ^{2} \Omega-\sin ^{4} \Omega\right)
\end{aligned}
$$

Where:

$\mathrm{q}_{\mathrm{w}, \mathrm{i}}^{0}=$ The convective heat flux with zero angle of attack $\Omega=$ The angle between the normal vector to the hemisphere at a given point and the dynamic pressure direction (Fig. 4)

For space vehicles plunging into dense atmospheres with velocities close to the second cosmic velocity, one should in addition to convective heat fluxes take into consideration the radiative heat transfer from the compressible gas layer in the shock wave (Nikitin, 2006):

$$
\mathrm{q}_{\mathrm{w}, \mathrm{r}}=7,845 \cdot \mathrm{R}\left(\frac{\rho}{\rho_{0}}\right)\left(\frac{\mathrm{V}}{1000}\right)^{8}\left[\frac{\mathrm{kW}}{\mathrm{m}^{2}}\right]
$$

Ablative Thermal Protection Coating (TPC) is considered as one of the most efficient and widespread means of protection of descent vehicles from impinging high-temperature heat flows (Nikitin, 2006; Duffa, 2013). In this case, the energy of external heat flows is spent on melting, evaporation and sublimation of coating components. To assess the mass of the entrained material of the ablative heat-protection coating it is convenient to use the formula (Nikitin, 2006):

$$
\dot{\mathrm{m}}_{\mathrm{w}}=\frac{\mathrm{q}_{\mathrm{w}, \mathrm{S}}}{\gamma\left(\mathrm{I}_{0}-\mathrm{I}_{\mathrm{w}}\right)+\mathrm{I}_{\mathrm{p}}}\left[\frac{\mathrm{kg}}{\mathrm{m}^{2} \mathrm{~s}}\right]
$$

Where:

$\dot{\mathrm{m}}_{\mathrm{W}}=$ The TPC mass-loss rate

$\gamma=$ The injection parameter

$\mathrm{I}_{0} \quad=$ The flow stagnation enthalpy

$\mathrm{I}_{\mathrm{w}}=$ The enthalpy on the vehicle surface

$I_{p}=$ The enthalpy of destruction or heat-destruction of the polymer

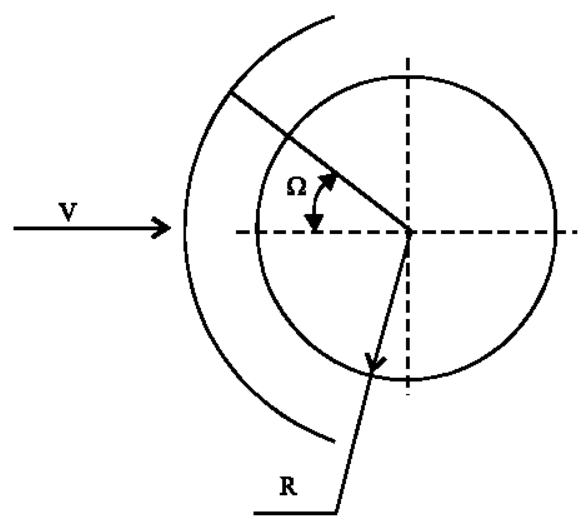

Fig. 4: Finding the convective heat flux density over the forward hemisphere

The injection parameter can be evaluated by the formula:

$$
\gamma=0.6 \cdot\left(\frac{I_{w}}{I_{0}}\right)^{0.03} \cdot\left(\frac{\mu_{e}}{\mu_{w}}\right)^{0.03}
$$

where, $\mu_{\mathrm{e}}$ and $\mu_{\mathrm{w}}$ are respectively, the mean molar-mass of components of the impinging flow and of the components of the injection gas.

The above TPC destruction model does not take into account the erosion effects and the effects related to carbonization and injection blocking (Nikitin, 2006; Eliseev and Tovstonog, 2014). For a composite polymer thermal protective material considered in the study we take $2200 \mathrm{k}$ (1200 k, respectively) as the mean destruction temperature of the filling (bonding) agent, the enthalpy destruction is taken to be $2000 \mathrm{~kJ} / \mathrm{kg}$.

Before proceeding with the consideration of an off-nominal descent with multiple plunges into the atmosphere, we consider the case of a nominal landing. As distinct from the case of Soyuz or Apollo type vehicles, the descent of ballistic vehicles (especially of those with near-parabolic velocity) features abrupt dives into the atmosphere which result in considerable thermal loads and accelerations acting on the vehicle. For a BC under consideration, for a direct (single) entrance into the atmosphere, the mass of the entrained thermal protection is estimated to be $195 \mathrm{~kg}$. Besides, the stationary temperature on the forward hemisphere when evaluated without consideration of TPC destruction, exceeds considerably (by 1.8 times) that of the TPC filling agent (which is taken to be $2200 \mathrm{k}$ ) (Fig. 5). For thermal analysis, the atmosphere entry altitude is taken to be $100 \mathrm{~km}$.

However, if the real destruction of the heat-protection cover is taken into account then a consequence of such an intense heating is that the heat temperature will not exceed the destruction temperature due to ablation. 


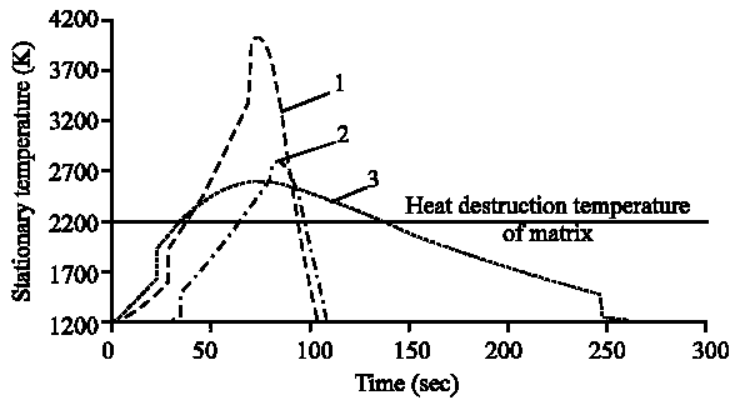

Fig. 5: Variation of the stationary temperature at vehicle's critical point versus time without consideration of TPC destruction (the results are given only for the interval with intensive TPC destruction); 1) A direct entry with second cosmic velocity; 2) A direct entry with first cosmic velocity and 3) Braking in the atmosphere during one entry (66.5 $\mathrm{km}$ perigee) with subsequent transfer to an ellipse of $200 \mathrm{~km}$ apogee altitude

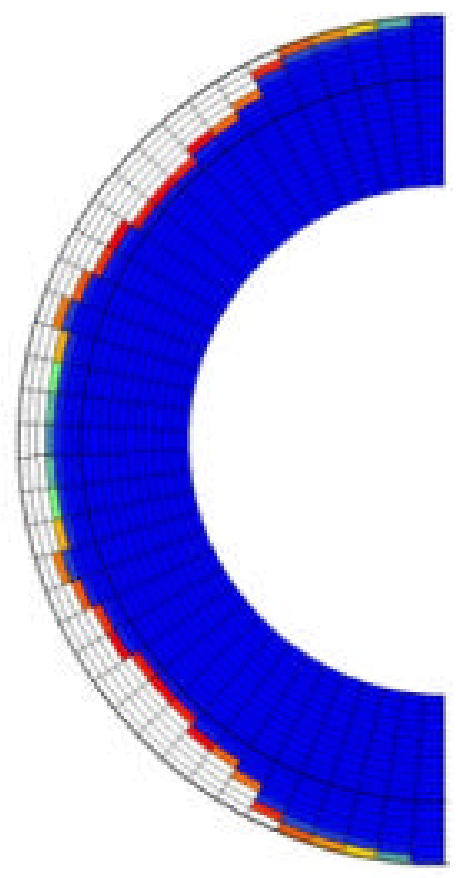

Fig. 6: Variation of the form of the forward hemisphere of a spherical $\mathrm{BC}$ with a direct entry into the dense atmosphere.

Besides, the form of the vehicle will vary through the descent (Fig. 6) due to active entrainment of TPC including the filling agent.

A preliminary deceleration to a near first cosmic velocity enables one to reduce the mass of the entrained TPC to $92 \mathrm{~kg}$. As a result, the mass and thickness of the entire thermal cover is reduced but a powerful retro engine

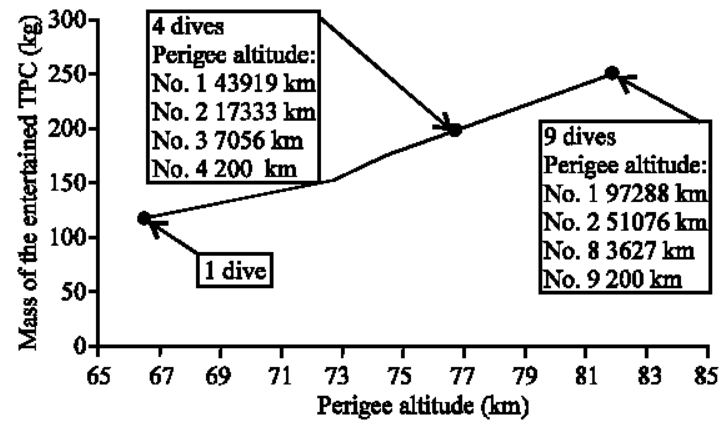

Fig. 7: Mass variation of the entrained TPC versus the perigee altitude of the return trajectory with repeated dives into the atmosphere (with a subsequent transfer to an ellipse of $200 \mathrm{~km}$ apogee)

is required to realize such a direct entry. In this case, the temperature will not exceed so much (and for a smaller time interval) the filling agent destruction temperature (Fig. 5). Hence, the most of the TPC entrained mass is in the bonding agent and so no considerable variation of the vehicle form ensues. A similar behavior is also observed for descents of Soyuz-type vehicles where the mean mass loss is $9 \mathrm{~kg} / 1 \mathrm{~m}^{2}$, the cover thickness reducing only by several millimeters. Figure 5 also shows the mass variation of an ablative TPC entrained from the forward hemisphere of a $\mathrm{BC}$ for the above entry trajectory with perigee of 66.5 $\mathrm{km}$ and a subsequent transfer to an ellipse with apogee of $200 \mathrm{~km}$.

With increasing number entries into the atmosphere, the vehicle considerably reduces its velocity during a small time when it passes the perigee values (entries into the atmosphere). Because of this, the intensity of the heat flows decreases from an entry to an entry which results in a smaller heat temperature of the surface. However, under this approach the heating effect is longer in time. Hence, the most of the TPC entrained mass is in the filling agent. The variation of the total mass of an ablative TPC entrained from the $\mathrm{BC}$ forward hemisphere during its repeated breaking in the upper atmosphere (with subsequent transfer to an ellipse of $200 \mathrm{~km}$ perigee altitude) is shown in Fig. 7 as a function of the perigee altitude of the return trajectory.

The further descent of the BC from the $66.5 \times 200 \mathrm{~km}$ elliptic orbit without any preliminary adjustment of the atmosphere entry parameters may result in a more flat trajectory in comparison with the classical descent profile from a near-circular orbit of altitude $200 \mathrm{~km}$ (Fig. 8). A similar situation may also appear if the perigee altitude of the return trajectory exceeds $66.5 \mathrm{~km}$. 


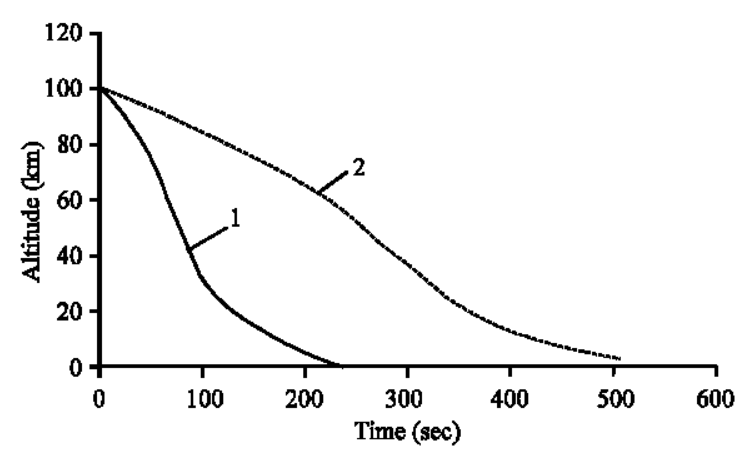

Fig. 8: BC descent trajectories; 1) A direct entry from a circular orbit and 2) The final stage after one intermediate dive with $66.5 \mathrm{~km}$ perigee altitude

Such a trajectory features a longer duration which results in a less intense heating (but for a longer time). The TPC entrainment will be about $100 \mathrm{~kg}$, its total value during the entire descent (with one intermediate dive) is $218 \mathrm{~kg}$.

The above graphs show that for a preliminary deceleration of the vehicle in the atmosphere to a local circular velocity by using repeated entries in the atmosphere with subsequent landing, the total TPC entrainment will be larger than for a direct descent, the most of the TPC entrained mass being in the bonding agent due to less intense heat flows. This fact limits the application of a number of materials in TPC, for example, asbestos-reinforced laminates for which the erosion resistance drastically decreases with removal of the bonding agent (Riccioa et al., 2017; Duffa, 2013; Eliseev and Tovstonog, 2014).

\section{CONCLUSION}

A near-Earth orbit with various apogee altitudes can be obtained by decelerating the descent module in the atmosphere. An atmosphere entrance with small perigee altitude gives a near circular orbit with smallest flight time but under this approach a BC (Ballistic Capsule) should be capable of withstanding considerable thermal loads. An increase of the perigee altitude of the return trajectory gives an elliptic orbit which rapidly degrades with decreasing eccentricity because its perigee lies in the dense atmosphere. The thermal loads acting on the vehicle decreases with larger initial perigee altitudes but their duration increases.

An increase in the duration of the exposure to high-temperature heat flows results in an increased mass of the entrained Thermal Protection Coating (TPC) but due to smaller temperatures the most of the TPC entrained mass is in the bonding agent.
The TPC degradation due to the entrainment of the bonding agent should be taken into account for the approaches involving repeated thermal loads of the vehicle (especially during the final descent to the Earth). The resistance of TPC materials against repeated sequential high-temperature heatings on the atmosphere segment and cooling on the orbital segment requires special analysis.

\section{ACKNOWLEDGEMENT}

The research was supported by Russian Science Foundation (project No. 17-79-10450)

\section{REFERENCES}

Avduevskii, V.S., 1992. [Heat-Transfer Principles for Aircraft and Rocket-and-Space Equipment]. Mashinostroenie Publishers, Moscow, Russia, (In Russian).

Duffa, G., 2013. Ablative Thermal Protection Systems Modeling. AIAA, Reston, Virginia, USA., ISBN:9781624101717, Pages: 431.

Eliseev, V.N. and V.A. Tovstonog, 2014. [Heat Exchange and Thermal Testing of Materials and Structures of Aerospace Engineering Under Radiation Heating]. Moscow Bauman University Press, Moscow, Russia, (In Russian).

Grabois, M.R., 2011. Apollo: Learning from the past, for the future. Acta Astronaut., 68: 1353-1360.

Hohmann, W., 1925. [The Accessibility of the Celestial Bodies: Studies on the Space Problem]. R. Oldenbourg Publisher, Germany, Pages: 88 (In German).

Jin, S., S. Arivazhagan and H. Araki, 2013. New results and questions of lunar exploration from SELENE, Chang'E-1, Chandrayaan-1 and LRO/LCROSS. Adv. Space Res., 52: 285-305.

Keller, J.W., N.E. Petro and R.R. Vondrak, 2016. The lunar reconnaissance orbiter mission-six years of science and exploration at the Moon. Icarus, 273: 2-24.

Lyndon, B.J., 1975. Apollo Program Summary Report. Johnson Space Center, Houston, Texas, USA., Pages: 508 .

Murtazin, R., 2014. Rendezvous missions: From ISS to lunar space station. Acta Astronaut., 101: 151-156.

Nikitin, P.V., 2006. [Thermal Protection]. Moscow Aviation Institute Press, Moscow, Russia, (In Russian).

Riccio, A., F. Raimondo, A. Sellitto, V. Carandente and R. Scigliano et al., 2017. Optimum design of ablative thermal protection systems for atmospheric entry vehicles. Appl. Therm. Eng., 119: 541-552. 